\title{
Electrocardiographic Finding
}

National Cancer Institute

\section{Source}

National Cancer Institute. Electrocardiographic Finding. NCI Thesaurus. Code C41328.

Graphic representation and categorization of the electrical vectors produced by the depolarization and repolarization of myocardial tissue. 